\title{
Schizanthus grahamii and Schizanthus hookeri. Is there any relationship between their anthocyanin compositions and their different pollination syndromes?
}

\author{
C. Alcalde-Eon ${ }^{\text {a }}$, J.C. Rivas-Gonzalo a , O. Muñoz ${ }^{\text {b }}$, M.T. Escribano-Bailón ${ }^{\text {a,* }}$ \\ ${ }^{a}$ Grupo de Investigación en Polifenoles (GIP), Unidad de Nutrición y Bromatología, Facultad de Farmacia, Universidad de Salamanca, Campus Miguel de Unamuno, \\ E-37007 Salamanca, Spain \\ ${ }^{\mathrm{b}}$ Departamento de Química, Facultad de Ciencias, Universidad de Chile, Casilla 653, Santiago, Chile
}

\section{A R T I C L E I N F O}

Article history:

Received 23 May 2012

Received in revised form 24 September 2012 Available online 25 October 2012

\section{Keywords:}

Schizanthus grahamii

Schizanthus hookeri

Solanaceae

HPLC-DAD-MS ${ }^{n}$

Anthocyanins

Hummingbird and bee-pollination

syndromes

\begin{abstract}
A B S T R A C T
Three different pollination syndromes (bee, hummingbird and moth-pollination syndromes) have been described for the different species of the genus Schizanthus Ruiz \& Pav. (Solanaceae). Two closely related species from a phylogenetic point of view, Schizanthus grahamii and Schizanthus hookeri, show hummingbird and bee-pollination syndromes, respectively. One of the traits used to assign these pollination syndromes is the colour of the petals, which is red in S. grahamii and bluish-pink in S. hookeri. The objective of this work was to establish the differences in the anthocyanin composition of these two Schizanthus species that contribute to the different pollination syndrome. Identification of the pigments was carried out from the chromatographic and spectral data supplied by the HPLC-DAD-MS ${ }^{n}$ analyses of the samples. Alkaline and acid hydrolyses were also performed in the extracts and in some isolated compounds in order to confirm the identities. First difference between these two species was found in the total anthocyanin content, which was about 13-fold higher in S. grahamii than in S. hookeri. Furthermore, the major peak was also different in both cases, thus explaining quantitative and qualitative colour differences between species. Delphinidin 3-O-rutinoside represented ca. $72 \%$ of the total area in S. grahamii and petanin (Petunidin 3-O-p-coumaroylrutinoside-5-O-glucoside) accounted for almost $62 \%$ of the total area in $S$. hookeri. The presence of the $p$-coumaroylrutinosyl moiety in the petanin makes the intramolecular copigmentation possible, thus conferring the bluish-pink hue to the petals of S. hookeri. Delphinidin 3$O$-rutinoside is in turn responsible for the red colour exhibited by the petals of S. grahamii and along with other floral traits, responsible for the attraction of hummingbirds to the plant. Pigments structurally related to petanin, which have been often detected in other genus of Solanaceae, were detected in both species and in similar contents, supporting results from previous studies that pointed to a bee-pollinated common ancestor for both species that further specialised acquiring traits attractive to hummingbirds and among them, red colour of the petals which is mainly supplied by delphinidin 3-O-rutinoside.
\end{abstract}

(c) 2012 Elsevier Ltd. All rights reserved.

\section{Introduction}

Flower phenotype is strongly conditioned by the mechanism of pollination. Whereas wind-pollinated flowers usually gather in catkins with inconspicuous or inexistent petals and do not produce nectar, flowers pollinated by animals present a suite of traits aimed at attracting them. This combination of features that differentially attracts one type of pollinator or another is known as pollination syndrome. Colour of the corolla is one of these traits and it can be due to the single accumulation of flavonoids (including anthocyanins), carotenoids or betalains or to the co-existence of more than one type of pigments. Betalains and flavonoids can co-exist with carotenoids but the co-existence between betalains and flavo-

\footnotetext{
* Corresponding author. Tel.: +34 923294537; fax: +34 923294515.

E-mail addresses: escriban@usal.es, a51711@usal.es (M.T. Escribano-Bailón).
}

noids has not been demonstrated yet (Harborne and Grayer, 1994; Miller et al., 2011; Mol et al., 1998). Among those pigments, anthocyanins are the major flower pigments in higher plants (Strack and Wray, 1994) and they are responsible for the red, pink or blue colour of the corollas. Modifications in the anthocyanin structure lead to different colour expression. Thus, the presence of one or another anthocyanin in the flower might affect the type of pollinators visiting the plant. In fact, colour preferences are different for different pollinators and it is known that bees prefer blue- and yellowflowered species, although they can also detect UV radiation, whereas birds visit red flowers (Harborne and Grayer, 1994; Miller et al., 2011). Although corolla colour is one of the most important signals in attracting pollinators (Miller et al., 2011; Wilbert et al., 1997) other signals as corolla shape, presence or absence of scent or the existence of nutritional rewards such as pollen or nectar will also determine the pollinator's choice. In fact, it is known that the pollinator can directly condition the acquisition of a given 
pollination syndrome by a plant (Pérez et al., 2006) and some examples of different species of a same genus showing different pollination syndromes have been reported (Harborne and Smith, 1978; Wilbert et al., 1997). The genus Schizanthus Ruiz \& Pavón (Solanaceae) is one of these genus showing different pollination syndromes. This genus is endemic to the southern South America Andean Region (Chile and Argentina) and comprises 12 species with three pollination syndromes: bee, hummingbird and mothpollination syndromes (Pérez et al., 2006). The floral morphology of this genus is unusual among the members of the Solanaceae and it reminds papilionaceous flower (Pérez et al., 2006). Colour of the corolla is one of the features used to classify one species into one pollination syndrome. Species presenting moth-pollination syndrome are white, those presenting bee-pollination syndrome are pink-purple and those showing a hummingbird-pollination syndrome are red (Pérez et al., 2006). From a phylogenetic point of view, Schizanthus grahamii Gill. ex Hook. and Schizanthus hookeri Gill. ex Graham (from now onwards S. grahamii and S. hookeri) are very closely related species and form a clade not only if genetic or morphologic features are considered (Pérez et al., 2006, 2011) but also when alkaloid composition is taken into account (Peña and Muñoz, 2002). Despite this, S. grahamii and S. hookeri show different pollination syndromes, hummingbird-pollination syndrome in the first case and bee-pollination syndrome in the second. This means that some of the floral traits are different in both species and among them the colour of the petals, that is red and bluishpink, respectively.

Although the alkaloid compositions of these species have been studied in detail (Cretton et al., 2011; Peña and Muñoz, 2002) there are no reports on their anthocyanin compositions. Thus, the objective of the present study was to establish the anthocyanin composition of these two related Schizanthus species in order to identify the differences that are responsible for the differences in the colour and that, consequently, contribute to the different pollination syndromes they show.

\section{Results and discussion}

During the extraction of the pigments from the petals differences in the colour of the extracts were quite noticeable. The extracts of $S$. grahamii were more intense in colouration than those of $S$. hookeri. Moreover, differences in the hue of the extracts were also observed, being those of the former redder than those of the latter. The results of the chromatographic analyses (Fig. 1) were in line with this observation. First, the total area at $520 \mathrm{~nm}$ of $S$. grahamii extracts was about 19-fold higher than those of S. hookeri, thus explaining the differences observed in the colour intensities of the extracts. Second, the major peaks were different in both cases (Fig. 1), being responsible for the differences observed in the hue. The chromatogram of $S$. grahamii (Fig. 1a) showed a major peak (72\% of the total area) at $17 \mathrm{~min}$ whereas in the case of $\mathrm{S}$. hookeri no important peaks, from a quantitative point of view, were observed before $36-37 \mathrm{~min}$. It is within this time range where the major peak (62\% of the total area) in the chromatogram of $S$. hookeri was located. When this part of the chromatogram was observed in detail in S. grahamii it could be seen that this peak, and those accompanying it, was also present and with similar areas (Fig. 1a inset). The mass spectrometry analyses revealed that the compounds eluting in these late peaks were the same in both species. The mass spectrometry analyses also allowed the detection of the major peak of S. grahamii in S. hookeri samples, but, as will be discussed later, in very low concentrations in relation to that determined in S. grahamii. Thus, although the major peaks were different in both species the mass spectrometry analyses showed that the samples were similar from a qualitative point of view: all the
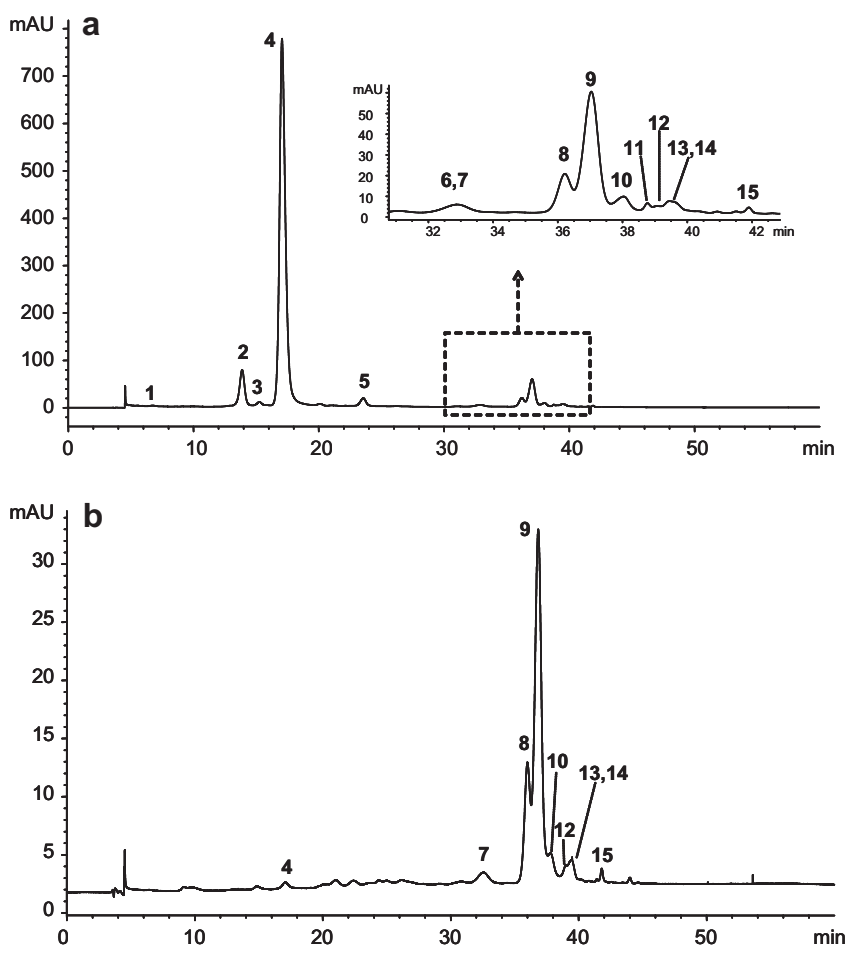

Fig. 1. Chromatograms recorded at $520 \mathrm{~nm}$ for (a) S. grahamii and (b) S. hookeri. Compound identities are listed in Table 1.

nine compounds detected in S. hookeri were also present in S. grahamii, but the latter possessed six additional compounds.

\subsection{Compound identification}

Table 1 shows the chromatographic and spectral features of the compounds detected in the samples as well as the identities proposed for them. As can be seen in the chromatogram recorded at $520 \mathrm{~nm}$ (Fig 1a), peak 4 was the major peak in S. grahamii. Its UV-vis spectrum showed three maxima at 526, 277 and $346 \mathrm{~nm}$ and was similar to those of the monoglucosides of the B-ring trisubstituted anthocyanidins, with a shoulder at $440 \mathrm{~nm}$ that was indicative of the absence of substitution in the 5-hydroxyl group (Harborne, 1958). The retention time was close to that of delphinidin 3-glucoside when analysed in similar conditions (Alcalde-Eon et al., 2004a, 2006). Nevertheless, the $m / z$ ratio of the molecular ion was not 465 but 611 . The fragmentation of this ion originated two major ions at $m / z 303$ (100 rel. int.) and $m / z 465$ (25) corresponding, respectively, to delphinidin and to a monohexoside of delphinidin. The ion of the monohexoside of delphinidin was originated by the loss of $146 \mathrm{amu}$, which might correspond to the loss of one rhamnosyl moiety or to the loss of one $p$-coumaroyl moiety. The absence of an additional peak at 309-312 nm in the UV-vis spectrum, characteristic of the $p$-coumaric acid, indicated that this residue corresponded to rhamnose. Thus, compound 4 was a delphinidin derivative containing a rhamnose linked to a hexose and linked to the anthocyanidin at the 3-position. Taking into account previous reports on the anthocyanin composition of different plants belonging to the Solanaceae family (Andersen and Jordheim, 2006), the hexose is likely to be glucose. In order to confirm the identities of the sugars present in compound $\mathbf{4}$, an acid hydrolysis was carried out on the isolated compound. The sugar-rich fraction was then analysed by HPTLC, and the identities of the sugars were established by comparison to standards of glucose, rhamnose and galactose. This analysis confirmed that compound $\mathbf{4}$ contained 
Table 1

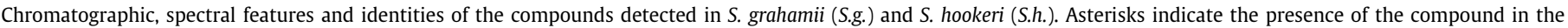
sample.

\begin{tabular}{|c|c|c|c|c|c|c|c|c|}
\hline Peak & $\begin{array}{l}t_{\mathrm{R}} \\
(\min )\end{array}$ & $\lambda_{\max }(\mathrm{nm})$ & $\begin{array}{l}\mathrm{M}^{+}(m / \\
z)\end{array}$ & MS $^{2}$ Frag. $m / z$ (relative intensity) & $\mathrm{MS}^{3}$ Frag. $m / z$ (relative intensity) & Compound identity & S.g. & S.h. \\
\hline 1 & 6.9 & & 773 & 611 (100), 303 (40), $465(10)$ & 303 (100), $465(40)$ & Dp-3-rut-7-glc & $*$ & \\
\hline 2 & 13.9 & $276,349,527$ & 773 & 303 (100), $627(7), 257(3), 465(1)$ & 257 (100), 229 (28), 303 (12) & Dp-3-glc-glc-rham & $*$ & \\
\hline 3 & 15.3 & & 465 & & & Dp-3-glc & $*$ & \\
\hline 4 & 17.1 & $277,346,526$ & 611 & 303 (100), 465 (25), 449 (3) & 257 (100), 229 (32), 303 (19) & Dp-3-rut & $*$ & $*$ \\
\hline 5 & 23.6 & 528 & 625 & $317(100), 479(19), 302(4), 463(2)$ & 302 (100), 257 (6), 317 (5) & Pt-3-rut & $*$ & \\
\hline 6 & 33 & & 949 & $\begin{array}{l}787(100), 317(85), 479(30), 625 \\
(1)\end{array}$ & $317(100), 479(15), 625(2)$ & Pt-3-cafrut-5-glc & $*$ & \\
\hline 7 & 33.2 & $279,314,531$ & 919 & 757 (100), 303 (35), 465 (35) & $303(100)$ & Dp-3-pcoumrut-5-glc & $*$ & $*$ \\
\hline 8 & 36.2 & 534 & 933 & $\begin{array}{l}771(100), 317(84), 479 \text { (38), } 609 \\
(5)\end{array}$ & $317(100), 479(14)$ & $\begin{array}{l}\text { Pt-3-pcoumrut-5-glc } \\
(Z)\end{array}$ & $*$ & $*$ \\
\hline 9 & 37 & $280,299,309,533$ & 933 & $\begin{array}{l}771(100), 317(92), 479 \text { (29), } 609 \\
(2)\end{array}$ & 317 (100), $479(6), 625(2)$ & $\begin{array}{l}\text { Pt-3-pcoumrut-5-glc } \\
(E)\end{array}$ & $*$ & $*$ \\
\hline 10 & 37.9 & $275,329,533$ & 963 & $\begin{array}{l}801 \text { (100), } 317 \text { (62), } 479 \text { (25), } 641 \\
(5)\end{array}$ & $\begin{array}{l}317(100), 479(22), 302(16), 625 \\
(2)\end{array}$ & Pt-3-ferrut-5-glc & $*$ & $*$ \\
\hline 11 & 38.8 & $\begin{array}{l}282,299,309,(440), \\
532\end{array}$ & 919 & $303(100), 627(15), 839(7)$ & 158 (100), 257 (90), 303 (50) & $\begin{array}{l}\text { Dp-3-glc-glc- } \\
\text { pcoumrham }\end{array}$ & $*$ & \\
\hline 12 & 39.1 & $280,299,309,535$ & 947 & $\begin{array}{l}785 \text { (100), } 331 \text { (76), } 493 \text { (13), } 623 \\
(11)\end{array}$ & 331 (100), 493 (12), $639(10)$ & $\begin{array}{l}\text { Mv-3-pcoumrut-5-glc } \\
(Z)\end{array}$ & $*$ & $*$ \\
\hline 13 & 39.5 & 282, 297, (310), 532 & 917 & $\begin{array}{l}755 \text { (100), } 301 \text { (82), } 798 \text { (70), } 463 \\
\text { (30) }\end{array}$ & $301(100)$ & Pn-3-pcoumrut-5-glc & $*$ & $*$ \\
\hline 14 & 39.6 & $279,297,312,533$ & 947 & 785 (100), 331 (75), 493 (19) & 331 (100), 493 (10), 639 (6) & $\begin{array}{l}\text { Mv-3-pcoumrut-5-glc } \\
(E)\end{array}$ & $*$ & $*$ \\
\hline 15 & 41.9 & $282,299,309,534$ & 771 & & & Pt-3-pcoumrut & $*$ & $*$ \\
\hline
\end{tabular}

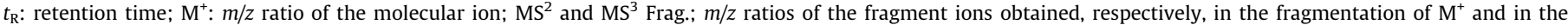

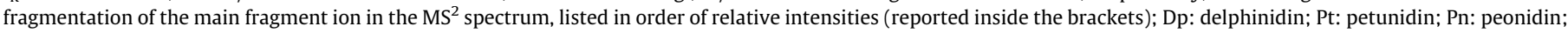
Mv: malvidin; Glc: glucose; Rut: rutinose; Rham: rhamnose; Caf: Caffeic acid; Pcoum: p-coumaric acid; Fer: ferulic acid.
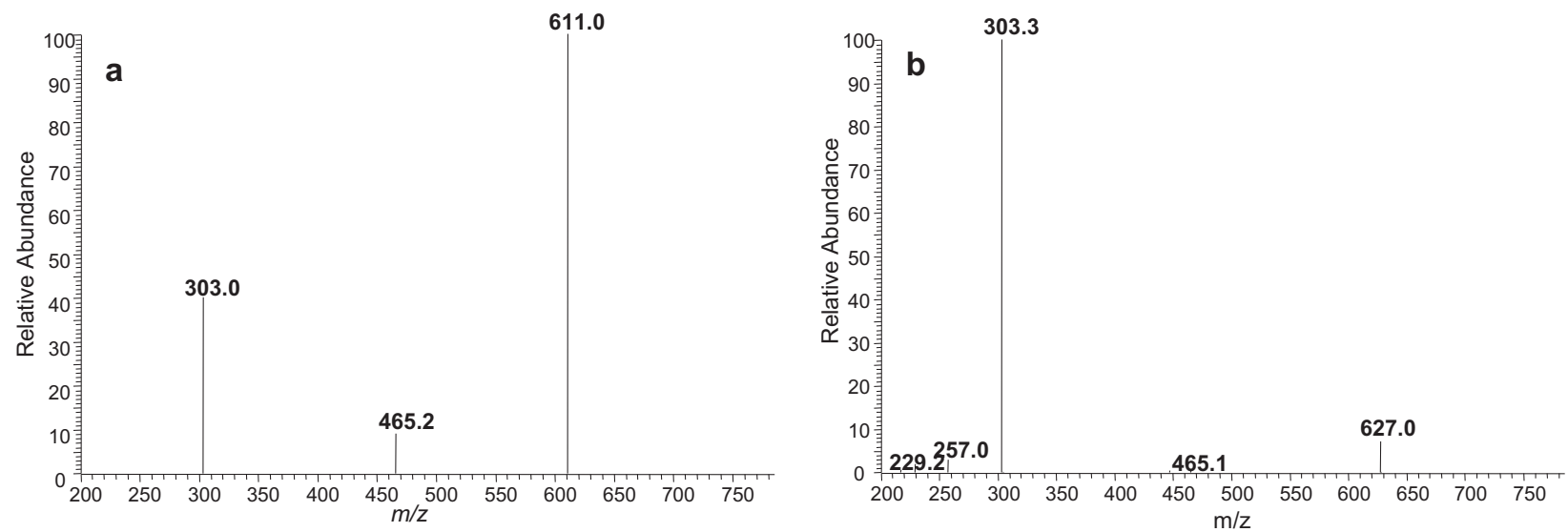

Fig. 2. $\mathrm{MS}^{2}$ spectra obtained from the fragmentation of the molecular ions ( $\mathrm{m} / \mathrm{z} 773$ in both cases) of compounds $\mathbf{1}$ (a) and $\mathbf{2}$ (b).

glucose and rhamnose. Galactose was not present. The positions involved in the linkage between the two sugar moieties could not be determined with the techniques here employed, but taking into account the substitution patterns in other members of the Solanaceae family (Rodríguez-Saona et al., 1998) and the substitution patterns of the rest of the pigments present in the Schizanthus samples that will be discussed later, compound $\mathbf{4}$ was identified as delphinidin 3-O-rutinoside.

Similarly, compound $\mathbf{5}$ that showed the same fragmentation pattern but with different anthocyanidin $(\mathrm{m} / \mathrm{z} 317)$ was identified as petunidin 3-O-rutinoside. This compound was only detected in S. grahamii.

The molecular ions of compounds $\mathbf{1}$ and $\mathbf{2}$ showed the same $\mathrm{m} / \mathrm{z}$ ratio $(m / z 773)$. Nevertheless, the fragmentation pattern was quite different (Fig. 2). As can be seen, both compounds contained delphinidin $(\mathrm{m} / \mathrm{z} 303)$ as aglycone with substituents accounting for 470 amu. Their UV-vis spectra supplied some relevant aspects: the absence of maxima in the region of 310-330 nm was indicative of the absence of acylation with hydroxycinnamic acids and the presence of a hump around $440 \mathrm{~nm}$ was indicative of the absence of substitution in 5-position of the anthocyanidin. In the case of compound 1, the molecular ion was fragmented in the MS ${ }^{2}$ analyses into three fragment ions, producing signals in the $\mathrm{MS}^{2}$ spectra at $m / z 611, m / z 465$ (monoglucoside of delphinidin) and $m / z 303$ (delphinidin). The first one was originated by loss of $162 \mathrm{amu}$ (one glucose) and its $\mathrm{m} / \mathrm{z}$ ratio was coincident with that of compound 4, delphinidin 3-O-rutinoside. This ion was further fragmented in the $\mathrm{MS}^{3}$ analyses, yielding two major fragment ions at $m / z 303$ and $m / z 465$, the same as those originated in the fragmentation of compound 4 . In the case of compound 2 , the fragmentation of the molecular ion yielded a major fragment ion at $\mathrm{m} / \mathrm{z}$ 303 (loss of all the substituents) and other ions with relative abundances lower than 10\%. Among these minor ions the most abundant was that of $\mathrm{m} / \mathrm{z} 627$ originated by the loss of $146 \mathrm{amu}$ (rhamnose) and corresponding to the diglucoside of delphinidin. Thus, compounds $\mathbf{1}$ and $\mathbf{2}$ contained the same sugars (two glucoses 
and one rhamnose) but attached differently to the delphinidin. Considering these fragmentation patterns and the fact that the linkage between two glucoses is not cleaved in the conditions usually employed but that between rhamnose and glucose can be cleaved (Giusti et al., 1999), two positions have to be involved in compound 1 whereas all the sugars have to be attached to the same position in compound $\mathbf{2}$. Since, as indicated previously, the hydroxyl of 5-position has not to be substituted, sugars in compound 1 have to be in position 3 and 7. Taking into account the structure of compounds $\mathbf{4}$ and $\mathbf{5}$, in which the rutinose is linked to position 3, it was proposed that in the case of compound $\mathbf{1}$, rutinose was also in position 3 and that the additional glucose was in position 7. In compound $\mathbf{2}$, the substituent has to be rhamnosylglucosylglucoside, with the rhamnose moiety located at the end of the substituent, thus explaining the formation of fragment ion at $m / z$ 627. Compound 1 was identified as delphinidin 3$O$-rutinose-7-O-glucose and compound $\mathbf{2}$ as delphinidin 3$O$-rhamnosylglucosylglucoside. These two compounds were only detected in S. grahamii.

Compound 3 was identified as delphinidin 3-O-glucoside taking into account the $\mathrm{m} / \mathrm{z}$ ratio of the molecular ion $(\mathrm{m} / \mathrm{z} 465)$ and the retention time in comparison to the features of the standard. This compound was only present in S. grahamii.

Compound 9 was the major pigment in S. hookeri. From its UVvis spectrum it was possible to know that the compound was acylated with an hydroxycinnamic acid, probably $p$-coumaric acid, since the spectrum showed an additional peak in the UV region near the absorption maximum of that acid (310 nm). Furthermore, the spectrum indicated that the hydroxyl group of the 5-position was substituted, since no hump was observed at $440 \mathrm{~nm}$. Its molecular ion showed a signal at $m / z 933$ in the MS spectrum. This ion was fragmented in the $\mathrm{MS}^{2}$ analysis, producing three major fragment ions at $m / z 771, m / z 317$ and $m / z 479$, originated by the loss of 162 (one glucose moiety), 616 and $454 \mathrm{amu}$, respectively. The first fragment ion was fragmented in the $\mathrm{MS}^{3}$ analyses giving rise to the two others, by loss of 454 and $292 \mathrm{amu}$. The ion at $\mathrm{m} /$ $z 317$ indicated that compound $\mathbf{9}$ was a petunidin derivative and that at $m / z 479$, the presence of a second glucose moiety in the molecule. Taking into account the data supplied by the UV-vis spectra and the pattern of substitution of the other compounds detected, the loss of 292 amu can be attributed to the loss of a $p$-coumaric acid residue linked to a rhamnose moiety (146+ $146 \mathrm{amu})$. The results of the alkaline and acid hydrolyses confirmed the presence of $p$-coumaric acid, rhamnose and glucose and the absence of galactose. Thus, compound $\mathbf{9}$ can be identified as follows: petunidin 3-O-p-coumaroylrutinoside-5-O-glucoside (also known as petanin). The chromatographic and spectral features of this compound are identical to those of the main pigment found in the tubers of Solanum stenotomum L. (Alcalde-Eon et al., $2004 \mathrm{~b}$ ) which was identified as the $Z$-isomer of petunidin 3-O$p$-coumaroylrutinoside-5-O-glucoside based on the results of an isomerisation experiment and on the bibliographic data.

Compound $\mathbf{8}$ showed a signal in the mass spectrum at the same $m / z$ ratio $(m / z 933)$ as compound $\mathbf{9}$. Furthermore, the fragment ions produced in the MS ${ }^{2}$ and $\mathrm{MS}^{3}$ analyses were also the same as those obtained in the fragmentation of compound $\mathbf{9}$. Only slight differences in the relative abundances of some minor fragment ions could be observed (Table 1). Nevertheless, they were not enough to establish the structural differences between these two compounds. The UV-vis spectrum only supplied reliable data in the visible region, since a flavonol co-eluted with compound $\mathbf{8}$ modifying the absorption in the UV region. It was possible to determine the absorption maximum in the visible $(534 \mathrm{~nm})$, which was $1 \mathrm{~nm}$ higher than that of compound $\mathbf{9}$, and to observe the absence of a hump in the $440 \mathrm{~nm}$ region indicative of the substitution of the hydroxyl group in 5-position. From these data, the possibility of being position isomers (3,5 and 3,7 for example) was discarded. On the other hand, if the positions involved in the glycosidic linkage between the rhamnose and glucose were different for each compound, the fragmentation pattern would probably show more differences than those observed. Nevertheless, if the sugars involved were isomers of the glucose or rhamnose, no differences would be detectable neither in the UV-vis spectra nor in the fragmentation pattern. Most of the anthocyanins identified in plants of the Solanaceae family share the substitution pattern with glucose and rhamnose being the sugars involved (Andersen and Jordheim, 2006). However, galactose has been detected in small amounts in tubers of native Andean Solanum tuberosum L. cultivars (André et al., 2007). For this reason, alkaline and acid hydrolyses were also carried out in compound $\mathbf{8}$ in order to check the presence or absence of galactose in this compound. No spot was detected at the $\mathrm{Rf}$ of the galactose in the HPTLC analysis whereas those of glucose and rhamnose were detectable. Thus, the hypothesis that compounds $\mathbf{8}$ and $\mathbf{9}$ might differ in the hexose involved in each compound was also discarded. If, as previously indicated, peak 9 is considered to be the $Z$-isomer of petunidin 3-O-p-coumaroylrutinoside-5-O-glucoside, the possibility of being $Z-E$ isomers (peak $8, E$-isomer and peak $9, Z$-isomer) might also be ruled out, since $Z$-isomers usually elute before the corresponding $E$-isomers (Ichiyanagi et al., 2005; Tatsuzawa et al., 2000). The HPLC-DAD-MS analysis of the organic phase originated from the alkaline hydrolysis of the purified $S$. grahamii anthocyanin extract revealed the presence of a major peak whose retention time and UV spectrum were identical to those of the standard of $p$-coumaric acid. The $\mathrm{m} / \mathrm{z}$ ratio of the molecular ion was also the same as that of that acid, thus confirming that $p$-coumaric acid is the major acylating compound in the sample. However, only the $E$-isomer of $p$-coumaric acid is commercially available. This would mean that compound 9, the most abundant of the two isomers, should be substituted with the $E$-isomer of $p$-coumaric acid. If compound $\mathbf{8}$ were the $Z$ isomer of compound $\mathbf{9}$, then the presence of the $Z$-isomer of the $p$-coumaric acid should be also detected in the organic phase. In fact, in the chromatogram of the organic phase there was a peak eluting just before that corresponding to the $E$-isomer of the $p$-coumaric acid, which also showed the same $m / z$ ratio as it, but different UV spectrum. Since the $Z$-isomer of $p$-coumaric acid is not commercially available, a solution of the $E$-isomer standard $(1.5 \mathrm{mg} / \mathrm{mL}$, methanol:water 30:70) was placed in a quartz cuvette and irradiated with UV light ( $360 \mathrm{~nm}$ ) for $24 \mathrm{~h}$ in order to form the $Z$-isomer similarly to the protocols employed in the isomerisation of anthocyanins acylated with this acid (Alcalde-Eon et al., 2004b; George et al., 2001). This solution was then analysed by HPLCDAD-MS. Two peaks appeared in the chromatogram (recorded at $280 \mathrm{~nm}$ ), one corresponding to the $E$-standard and the other, which was originated from it, should correspond to the $Z$-isomer. The chromatographic and spectral features of this compound were the same as those of the peak observed in the chromatogram of the organic phase that eluted before the $E$-isomer, thus indicating that the $Z$-isomer of the $p$-coumaric acid was also taking part in the acylation of some of the pigments. The area of the peak corresponding to the E-isomer was ca. 4-fold higher than that of the $Z$-isomer, and this proportion is similar to that observed between peaks 8 and 9 in the $S$. grahamii extract before being hydrolysed. Taking into account these results peak 8 was identified as the $Z$ isomer of Pt-3-O-p-coumaroylrutinoside-5-O-glucoside and peak 9 as the E-isomer of this same compound, which is in accordance with the elution order observed for these kinds of compounds by other research groups (Ando et al., 1999; Ichiyanagi et al., 2005; Tatsuzawa et al., 2000. Consequently, the major compound found in the tubers of $S$. stenotomum should also correspond to the $E$-isomer. Some additional studies should be done in these tubers in order to establish what type of isomer it is. 
The fragmentation patterns observed in the fragmentation of compounds 7, 12, 13 and $14(m / z$ 919, 947, 917 and 947, respectively) were the same as that observed in compounds $\mathbf{8}$ and $\mathbf{9}$ (Table 1), thus pointing to related structures but with different anthocyanidins involved. In fact, in the fragmentation of these compounds, the presence of two glucose moieties, one rhamnose and one p-coumaroyl residue can be inferred from the losses observed in the $\mathrm{MS}^{2}$ and $\mathrm{MS}^{3}$ analyses and the identities of the anthocyanidins (delphinidin, malvidin, peonidin and malvidin, respectively), from the resulting ions $(\mathrm{m} / \mathrm{z} 303,331,301$ and 331). Compounds 7 and $\mathbf{1 3}$ were identified as the 3-O-pcoumaroylrutinoside-5-O-glucoside derivatives of delphinidin and peonidin, respectively. The case of compounds 12 and 14 was similar to that of compounds $\mathbf{8}$ and $\mathbf{9}$, both compounds showing molecular and fragment ions at identical $\mathrm{m} / \mathrm{z}$ ratios. Compound 14 was therefore identified as the E-isomer of malvidin 3-O-pcoumaroylrutinoside-5-O-glucoside and compound $\mathbf{1 2}$ as the $Z$-isomer of the same compound.

Compounds 6 and 10 showed molecular ions with $m / z$ ratios 16 and 30 amu higher than that of compound $\mathbf{9}$ sharing with it most of the fragment ions. In both cases, the molecular ion was fragmented in the $\mathrm{MS}^{2}$ analysis into a major ion $(\mathrm{m} / \mathrm{z} 787$ for compound $\mathbf{6}$ and $\mathrm{m} / \mathrm{z} 801$ for compound 10) by loss of one glucose moiety. The fragmentation of these molecular ions in the $\mathrm{MS}^{3}$ analyses yielded the same major fragment ions in both cases and the same as those obtained in the fragmentation of compound $\mathbf{9}$, i.e. the fragment ions corresponding to petunidin and to its monoglucoside. In the case of compound $\mathbf{9}$, the ion of the monoglucoside was originated from the ion at $m / z 771$ by the loss of 292 amu which was attributed to the loss of one $p$-coumaroyl residue linked to a rhamnose moiety. In the cases of compounds $\mathbf{6}$ and 10, this ion was originated from the ion at $m / z 787$ by the loss of 308 amu and from the ion at $m / z 801$ by the loss of 322 amu, respectively. Taking into account the substitution pattern of the other pigments present in the sample, the usual substitution pattern in the Solanaceae family and, in the case of compound 10, the presence of a hump in the UV-vis spectra at $329 \mathrm{~nm}$, the losses of 308 and 322 amu were attributed to the simultaneous loss of a rhamnose moiety and a caffeoyl residue or a feruloyl residue, respectively. In fact, in the organic phase from the alkaline hydrolysis of the purified S. grahamii anthocyanin extract, caffeic and ferulic acid were detected and identified by comparison with standards. Thus, compound 6 was identified as petundin 3-O-caffeoylrutinoside-5-O-glucoside and compound $\mathbf{1 0}$ as petunidin 3-O-feruloylrutinoside-5-O-glucoside. Whereas compound $\mathbf{1 0}$ was detected in both S. grahamii and $S$. hookeri, compound $\mathbf{6}$ was only detected in S. grahamii.

The molecular ion of compound $\mathbf{1 1}$ showed the same $m / z$ as compound 7 ( $m / z$ 919). Nevertheless, neither the fragment ions nor the fragmentation pattern were the same. The major fragment ion in the $\mathrm{MS}^{2}$ spectrum was that corresponding to delphinidin $(\mathrm{m} /$ $z 303$ ) and was originated by loss of all the substituents (616 amu). The second most abundant ion (15\%) in the $\mathrm{MS}^{2}$ spectrum showed a signal at $m / z 627$ by loss of 292 amu (one rhamnose and one $p$ coumaroyl residue) and corresponded to delphinidin linked to two glucose moieties $(303+162+162)$. No signal was observed at $\mathrm{m} / \mathrm{z}$ of the monoglucoside of delphinidin, indicating that both glucose moieties had to be linked. The UV-vis spectrum confirmed the presence of the $p$-coumaric acid in the compound since it showed a shoulder at $309 \mathrm{~nm}$. Furthermore, from this spectrum it was possible to know the absence of substitution at the 5position hydroxyl group of the delphinidin, since there was a hump in the $440 \mathrm{~nm}$ region. From all these data, compound 11 was identified as delphinidin 3-O-p-coumaroylrhamnosylglucosylglucoside, the coumaroylated derivative of compound 2 . The analysis of the aqueous phase obtained after the alkaline hydrolysis of the purified S. grahamii extract demonstrated the relationship between compounds 2 and 11, since the relative amount of compound $\mathbf{2}$ increased after the hydrolysis. As occurred for compound 2, compound $\mathbf{1 1}$ was only present in S. grahamii.

The last peak (15) in the chromatogram showed a signal in the mass spectrum at $m / z 771$, which is coincident with that of the ion originated by the loss of one glucose moiety in compounds 8 and $\mathbf{9}$. It was not possible to obtain its fragmentation in order to verify if compound $\mathbf{1 5}$ was the de-glucosylated derivative of compounds $\mathbf{8}$ or 9 but the information supplied by the UV-vis spectra was helpful: the presence of the hump in the $440 \mathrm{~nm}$ region indicated the absence of substitution in the hydroxyl group of $\mathrm{C} 5$ and the shoulder at $309 \mathrm{~nm}$, the presence of acylation with p-coumaric acid. Furthermore, these spectral features and the retention time were the same as those of petunidin 3-O-p-cumaroylrutinoside identified in S. stenotomum tubers (Alcalde-Eon et al., 2004b) and thus, this identity was assigned to compound $\mathbf{1 5}$. In addition, the slight increase in the relative content of compound $\mathbf{5}$ (petunidin 3-O-rutinoside) observed in the aqueous phase after the alkaline hydrolysis of the purified extract might support the identity proposed for compound 15. This compound was detected in both Schizanthus species.

Most of the compounds detected and identified in Schizanthus samples in this study had been already detected and identified in flowers and other organs from other genera of the Solanaceae family (Andersen and Jordheim, 2006) In fact, the substitution pattern (3-[6-(4-p-coumaroyl/caffeoyl/feruloyl-rhamnosyl)-glucoside]-5glucoside) of eight of the nine common compounds detected in both Schizanthus species (compounds 7, 8, 9, 10, 12, 13, 14 and $\mathbf{1 5}$ ) and that of compound $\mathbf{6}$, only detected in $S$. grahamii, has been reported to be the dominant one in flowers and/or tubers of the genera Petunia and Solanum (Ando et al., 1999; André et al., 2007; Fossen et al., 2003; Ichiyanagi et al., 2005). Taking into account our own results and the results of these previous studies, the structures for these typical Solanaceae pigments detected in Schizanthus are shown in Fig. 3. Furthermore, in order to confirm that these Schizanthus pigments were of this "Solanaceae" kind, a S. stenotomum extract, previously characterised in our laboratory (Alcalde-Eon et al., 2004b), was analysed employing the same conditions as those of the present study. The chromatographic and spectral (UV-vis and MS) features of the pigments of S. stenotomum were compared to those of these Schizanthus pigments, revealing that pigments $6,7,9,10,13,14$ and 15 were the same as most of those previously detected in the Andean tubers extract (Figure 4).

\subsection{Differences in the anthocyanin composition of S. grahamii and $S$.} hookeri. Relationship between composition and pollination syndrome

The qualitative and quantitative differences between the anthocyanins of both species can be observed in Table 2. This Table shows the percentage of the total area of each anthocyanin detected in each species as well as their individual concentration. The total anthocyanin concentration, calculated as a sum of the individual pigment concentration, is also reported.

From a qualitative point of view the main difference between both species was the presence in high amounts of delphinidin 3$O$-rutinoside (compound 4) in S. grahamii $(29.5 \mathrm{mg} / \mathrm{g}$ of dried petals) which was almost absent $(0.2 \mathrm{mg} / \mathrm{g})$ in $S$. hookeri. Furthermore, compounds 1, 2, 3 and 5, that have structures related to that of compound 4, and compound 11 that seems to be the coumaroylated derivative of compound $\mathbf{2}$ were only detected in S. grahamii and along compound 4 they accounted for more than $80 \%$ of the total area. On the contrary, the pigments structurally related to petanin (compounds 7-10 and 12-15) were present in both species and their contents were quite similar (Table 2). Nevertheless, their percentages over the total area were much higher in S. hookeri than in S. grahamii. In fact, in S. hookeri, compounds $\mathbf{8}$ and $\mathbf{9}$ accounted for 


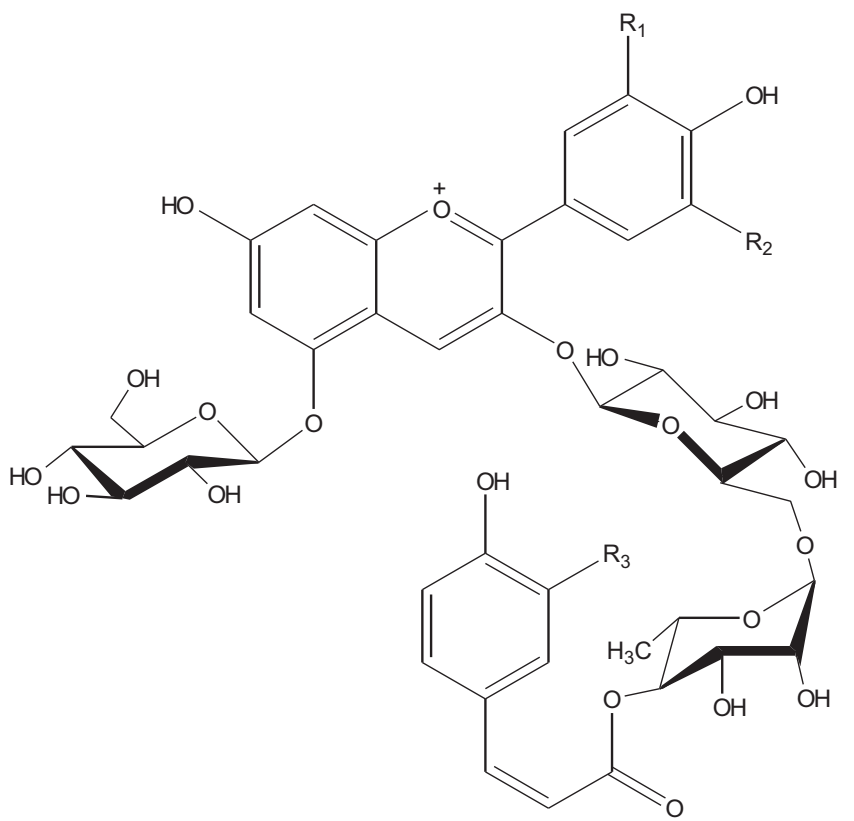

Z-isomers

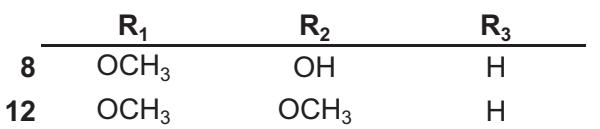

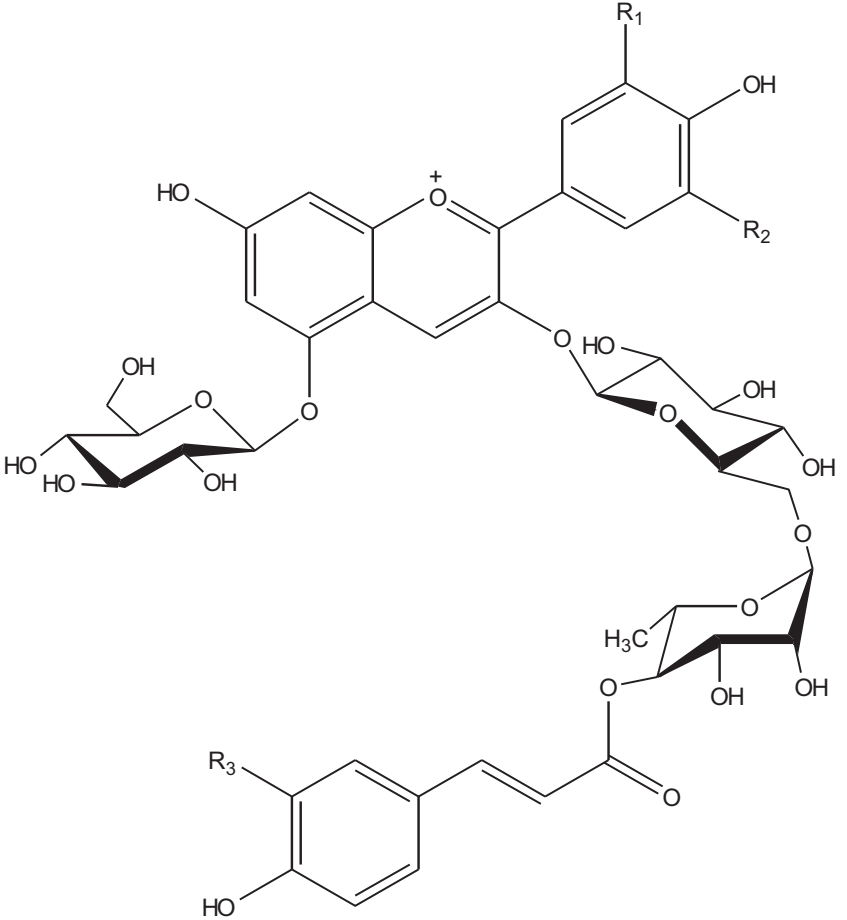

E-isomers

\begin{tabular}{rccc} 
& $\mathbf{R}_{\mathbf{1}}$ & $\mathbf{R}_{\mathbf{2}}$ & $\mathbf{R}_{\mathbf{3}}$ \\
\cline { 2 - 4 } $\mathbf{0}$ & $\mathrm{OCH}_{3}$ & $\mathrm{OH}$ & $\mathrm{OH}$ \\
$\mathbf{7}$ & $\mathrm{OH}$ & $\mathrm{OH}$ & $\mathrm{H}$ \\
$\mathbf{9}$ & $\mathrm{OCH}_{3}$ & $\mathrm{OH}$ & $\mathrm{H}$ \\
10 & $\mathrm{OCH}_{3}$ & $\mathrm{OH}$ & $\mathrm{OCH}_{3}$ \\
13 & $\mathrm{OCH}_{3}$ & $\mathrm{H}$ & $\mathrm{H}$ \\
14 & $\mathrm{OCH}_{3}$ & $\mathrm{OCH}_{3}$ & $\mathrm{H}$
\end{tabular}

Fig. 3. Structures of the Schizanthus pigments which possess a substitution pattern typical of Solanaceae.

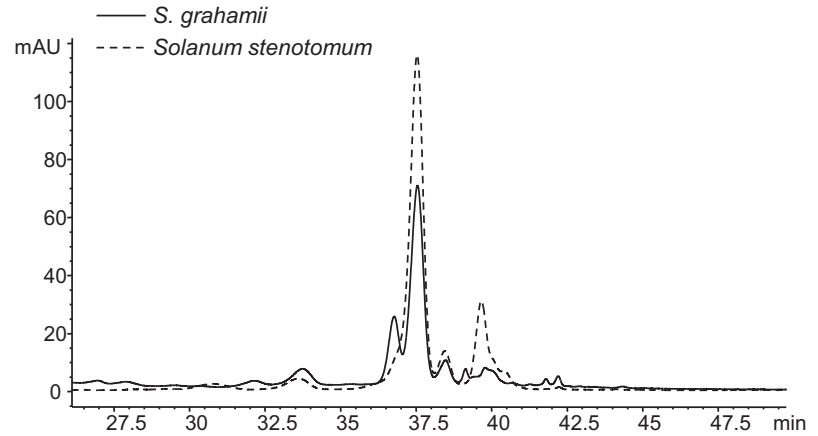

Fig. 4. Detail of the chromatograms obtained from Schizanthus grahamii petals (solid line) and Solanum stenotomum tubers (dotted line) recorded at $520 \mathrm{~nm}$ (time range 26-50 $\mathrm{min}$ ).

$80 \%$ of the total area ( $20.1 \%$ and $61.2 \%$, respectively). These differences in the major anthocyanins along with their different total anthocyanin contents $(38.6 \mathrm{mg} / \mathrm{g}$ in $S$. grahamii and $2.9 \mathrm{mg} / \mathrm{g}$ in $S$. hookeri) might explain the colour differences observed in the petals of both species: S. grahamii petals are red coloured and those of $S$. hookeri, light bluish-pink. In the second case, most of the colour is due to petanin and petanin-related pigments. In these pigments, the presence of the $p$-coumaroylrutinosyl moiety allows the molecule to adopt a folded structure, making the intramolecular copigmentation possible (Dangles et al., 1993). This conformation of the molecule protects the pigments against the hydration reaction and causes a batochromic shift in their visible absorption maximum and a hyperchromic effect, which is more remarkable in $Z$-isomers than in $E$-forms (George et al., 2001). This phenomenon might explain the bluish hue observed in the colour of the petals of $S$. hookeri in relation to the colour of the petals of S. grahamii. In the latter, the major anthocyanin is delphinidin 3-O-rutinoside, a nonacylated pigment in which the intramolecular copigmentation cannot take place. Although the presence of delphinidin in the petals of the flowers has been traditionally associated to blue colouration (Harborne and Grayer, 1994; Harborne and Smith, 1978; Saito and Harborne, 1992), most of the delphinidin related pigments identified in those studies possessed substituents which could make intramolecular copigmentation possible ( $p$-coumaroylrutinoside or $p$-coumaroylglucoside, for example). On the contrary, in recent studies carried out in the genus Petunia, which also belongs to the Solanaceae family, it has been demonstrated that flowers containing delphinidin 3-O-rutinoside were classified as red through different colorimetric determinations (Ando et al., 1999, 2004). Nevertheless, it has to be kept in mind that flower hue is not only determined by the amounts and type of anthocyanins accumulated by the flower. Other factors such as vacuolar $\mathrm{pH}$, temperature, age, presence of metals or cell shape can modify the colour expressed by the same pigments (Miller et al., 2011; Mol et al., 1998). For 
Table 2

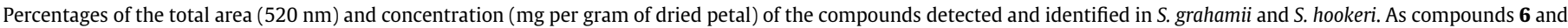

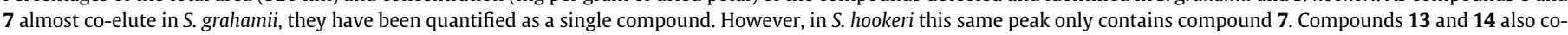
eluted and they have been quantified as a single compound.

\begin{tabular}{|c|c|c|c|c|c|}
\hline \multirow[t]{2}{*}{ Peak } & \multirow[t]{2}{*}{ Compound identity } & \multicolumn{2}{|c|}{$\%$ Of the total area } & \multicolumn{2}{|c|}{ C (mg/g dried petal) } \\
\hline & & S. grahamii & S. hookeri & S. grahamii & S. hookeri \\
\hline 1 & Dp-3-rut-7-glc & $0.1 \pm 0.01$ & & $0.2 \pm 0.01$ & \\
\hline 2 & Dp-3-glc-glc-rham & $7.1 \pm 0.46$ & & $3.1 \pm 0.34$ & \\
\hline 3 & Dp-3-glc & $1.5 \pm 0.16$ & & $0.8 \pm 0.09$ & \\
\hline 4 & Dp-3-rut & $72 \pm 2.06$ & $0.9 \pm 0.25$ & $29.5 \pm 1.15$ & $0.2 \pm 0.01$ \\
\hline 5 & Pt-3-rut & $2.4 \pm 0.23$ & & $0.9 \pm 0.10$ & \\
\hline $\begin{array}{l}6 \\
7\end{array}$ & $\begin{array}{l}\text { Pt-3-cafrut-5-glc } \\
\text { Dp-3-pcoumrut-5-glc }\end{array}$ & $0.9 \pm 0.18$ & $2.5 \pm 0.19$ & $0.5 \pm 0.08$ & $0.2 \pm 0.01$ \\
\hline 8 & Pt-3-pcoumrut-5-glc (Z) & $1.6 \pm 0.12$ & $20.1 \pm 0.44$ & $0.6 \pm 0.03$ & $0.5 \pm 0.03$ \\
\hline 9 & Pt-3-pcoumrut-5-glc $(E)$ & $4.9 \pm 0.43$ & $61.6 \pm 0.63$ & $1.5 \pm 0.10$ & $1.1 \pm 0.07$ \\
\hline 10 & Pt-3-ferrut-5-glc & $0.7 \pm 0.08$ & $4.4 \pm 0.19$ & $0.4 \pm 0.03$ & $0.3 \pm 0.01$ \\
\hline 11 & Dp-3-glc-glc-pcoumrham & $0.3 \pm 0.03$ & & $0.3 \pm 0.02$ & \\
\hline 12 & Mv-3-pcoumrut-5-glc $(Z)$ & $0.2 \pm 0.02$ & $2.1 \pm 0.13$ & $0.3 \pm 0.01$ & $0.2 \pm 0.01$ \\
\hline $\begin{array}{l}13 \\
14\end{array}$ & $\begin{array}{l}\text { Pn-3-pcoumrut-5-glc } \\
\text { Mv-3-pcoumrut-5-glc }(E)\end{array}$ & $0.5 \pm 0.05$ & $4.8 \pm 0.40$ & $0.4 \pm 0.02$ & $0.3 \pm 0.01$ \\
\hline \multirow[t]{2}{*}{15} & Pt-3-pcoumrut & $0.2 \pm 0.05$ & $1.3 \pm 0.17$ & $0.3 \pm 0.01$ & $0.2 \pm 0.01$ \\
\hline & & & Total & $38.6 \pm 1.66$ & $2.9 \pm 0.12$ \\
\hline
\end{tabular}

Dp: delphinidin; Pt: petunidin; Pn: peonidin; Mv: malvidin; Glc: glucose; Rut: rutinose; Rham: rhamnose; Caf: Caffeic acid; Pcoum: p-coumaric acid; Fer: ferulic acid.

example, flowers can become blue with age or if anthocyanins are complexed with different metals. In relation to $\mathrm{pH}$, hue can be shifted towards red if $\mathrm{pH}$ becomes more acidic or towards blue if it becomes more alkaline. Furthermore, differences in the behaviour of simple and more complex pigments towards $\mathrm{pH}$ changes have been reported (Fossen et al., 1998). In the present study, clear differences in the total pigment content and in the major type of anthocyanin have been detected between both species of Schizanthus. Thus, differences in the colour of the petals might be mainly attributed to the composition differences, although factors such as vacuolar $\mathrm{pH}$ or age of the plant might be enhancing or diminishing these colour differences.

The colour of the corolla is one of the traits that are used to ascribe animal-pollinated plants to one type of pollination syndrome or to another, but other features of the flower such as presence or absence of scent, nutritional rewards for the pollinator (nectar, pollen), shape and architecture of the inflorescence, etc. are also important to define the pollination syndrome. Pérez et al. (2006) assigned a hummingbird-pollination syndrome to $S$. grahamii and a bee-pollination syndrome to $S$. hookeri on the basis of eight different floral traits, and among them colour of the corolla, red in S. grahamii and light pink-purple in S. hookeri. Although some of their morphological characters are different, the phylogenetic studies on the genus Schizanthus have revealed that these two species belong to the same clade (Peña and Muñoz, 2002; Pérez et al., 2006). Furthermore, the ancestral reconstruction of flower visitors carried out on this genus (Pérez et al., 2006) revealed that the ancestral flower was probably bee-pollinated, and that the traits associated with hummingbird-pollination, red corollas among them, evolved only in S. grahamii. This transition from bee-pollination to hummingbird-pollination seems to occur in environments with low insect activity, as can be the high elevations in the central Chilean Andes at which these species grow (Pérez et al., 2006). The results obtained in the present study on the anthocyanin composition of these two species of the genus Schizanthus are in line with those previous reports. As indicated previously and as can be seen in Table 2, the petanin-related pigments that are the major pigments in the bee-pollinated $S$. hookeri are also present in S. grahamii and in very similar contents. This might be related to the fact of possessing a bee-pollinated common ancestor. The later specialisation of $S$. grahamii on hummingbirdpollination with the acquisition of floral traits attractive to these pollinators (Pérez et al., 2007) would have promoted, among others, the synthesis of delphinidin 3-O-rutinoside and related pig- ments, only detected in S. grahamii. In fact, transition from anthocyanins having complicated side residues (petanin for example) to simpler anthocyanins (delphinidin 3-O-rutinoside for instance) has also been observed in the evolution of wild taxa of Petunia (Ando et al., 1999), another genus belonging to Solanaceae. Furthermore, these "red" and simpler pigments were present in $S$. grahamii in much higher contents than the petanin-related ones thus explaining the change in petals colour from light bluish-pink to red. This quantitative difference in the pigment content between hummingbird-pollinated and bee-pollinated species has also been observed in related species from other genera and families (Wilbert et al., 1997). The lower pigment content in S. hookeri is related to the bee-pollination syndrome that it bears. Most of bees are attracted by blue and yellow flowers and have been traditionally considered to be insensitive to red colour (Harborne and Grayer, 1994). This is not strictly true and they can perceive red objects (Rodríguez-Gironés and Santamaría, 2004). However, it seems that it is more difficult for bees to find red flowers than blue flowers since they cannot easily discriminate red from a green background such as foliage. Thus, bees seem to be more attracted by blue than by red flowers because they require less time to find them (Rodríguez-Gironés and Santamaría, 2004). Blue hue due to the intramolecular copigmentation taking place in the petanin and petaninrelated pigments might play, along with other floral traits, a major role in the attraction of bees to $S$. hookeri. However, in bee-pollinated flowers not only colour is important by itself but also its patterning and the presence of UV-absorbing compounds, since bees are able to see in UV light (Harborne and Grayer, 1994; Miller et al., 2011). Thus, in order to be attractive to their pollinators bee-pollinated flowers do not need pigment contents as high as hummingbird-pollinated flowers do. In addition, colour in S. hookeri seems not to be as relevant in the attraction of bees to the flower as the red colour is in the attraction of hummingbirds to $S$. grahamii. S. grahamii specialised and synthesised red pigments, whereas $S$. hookeri maintained an anthocyanin profile typical of Solanaceae. In spite of possessing a bee-pollination syndrome, $S$. hookeri seems to be a generalist species. Pérez et al. (2006) reported that bees were not the only visitors of S. hookeri flowers, but also flies and hummingbirds. Some unspecialized flies seem to be attracted by similar pigments as bees (Harborne and Grayer, 1994) and in the case of hummingbirds colour of the corolla of $S$. hookeri is not the trait responsible for the attraction, but is the high nectar production. The stressful conditions of the high Andes region where these two species grow affect pollinator availability. 
Pérez et al. (2009) have suggested that S. hookeri and S. grahamii have evolved following two different pathways. S. hookeri has opted for generalised pollination. Thus, the broader range of possible pollinators compensates for the fluctuations in pollinator abundance. On the contrary, it seems that specialisation of $S$. grahamii on hummingbirds have been accompanied by the evolution of delayed autonomous selfing. This strategy assures seed set in times of pollinator scarcity.

\section{Concluding remarks}

The anthocyanin compositions of the petals of S. grahamii and $S$. hookeri have been reported for the first time in the present study. These two phylogenetically related species first differ in the total anthocyanin content, which is much higher in S. grahamii than in S. hookeri. Furthermore, their major anthocyanins are different: in $S$. grahamii, delphinidin 3-O-rutinoside (red) is the major one whereas in S. hookeri, the $Z$ - and $E$-isomers of petanin (bluish-pink) account for more than $80 \%$ of the total area. These qualitative and quantitative differences are in accordance with the pollination syndromes ascribed to them, hummingbird-pollination syndrome for S. grahamii and bee-pollination syndrome for S. hookeri. In addition, the existence of common pigments in both species and in similar contents supports the results of previous phylogenetic studies that pointed to a common ancestor of these two species. The synthesis of structurally simpler red pigments by $S$. grahamii seems to be related to the further specialisation of this species to attract hummingbirds.

\section{Experimental}

\subsection{General procedures}

HPLC-DAD analyses were performed in a Hewlett-Packard 1100 series liquid chromatograph. An AQUA C18 reversed-phase, $5 \mu \mathrm{m}$, $150 \times 4.6 \mathrm{~mm}$ column (Phenomenex ${ }^{\circledR}$, Torrance, CA, USA) thermostatted at $35{ }^{\circ} \mathrm{C}$, was used.

The HPLC-DAD conditions had been previously employed with satisfactory results in our laboratory in the analysis of other plant materials (tubers, grapes) (Alcalde-Eon et al., 2004a,b). The solvents used were: (A) an aq. soln. (0.1\%) of trifluoroacetic acid (TFA) and (B) 100\% HPLC-grade acetonitrile, establishing the following gradient: isocratic $10 \%$ B for $5 \mathrm{~min}$, from $10 \%$ to $15 \%$ B for $15 \mathrm{~min}$, isocratic $15 \%$ B for $5 \mathrm{~min}$, from $15 \%$ to $18 \%$ B for $5 \mathrm{~min}$ and from $18 \%$ to $35 \%$ B for $20 \mathrm{~min}$, at a flow rate of $0.5 \mathrm{~mL} / \mathrm{min}$. Detection was carried out at $520 \mathrm{~nm}$ as the preferred wavelength. Spectra were recorded from 220 to $600 \mathrm{~nm}$.

The mass spectrometry analyses were performed using a Finnigan $^{\mathrm{TM}}$ LCQ ion trap instrument (Thermoquest, San Jose, CA, USA) equipped with an API source using an electrospray ionisation (ESI) interface. The LC system was connected to the probe of the mass spectrometer via the UV-cell outlet. Nitrogen was used as sheath and auxiliary gas. The sheath gas flow was $1.2 \mathrm{~L} / \mathrm{min}$ and the auxiliary gas flow, $6 \mathrm{~L} / \mathrm{min}$. The capillary voltage was $4 \mathrm{~V}$ and the capillary temperature $195^{\circ} \mathrm{C}$. The source voltage was $4.5 \mathrm{kV}$. Spectra were recorded in positive ion mode between $\mathrm{m} / \mathrm{z} 120$ and 1500 . The mass spectrometer was programmed to do a series of three consecutive scans: a full mass, an $\mathrm{MS}^{2}$ scan of the most abundant ion in the full mass and an $\mathrm{MS}^{3}$ of the most abundant ion in the $\mathrm{MS}^{2}$. The normalised energy of collision was $45 \%$.

\subsection{Plant material}

Flowers of S. grahamii Gill. ex Hook. and S. hookeri Gill. ex Graham (S. grahamii and S. hookeri) were collected at Rengo (VI Region, O'higgins Province, Chile). Collection and identification were carried out by Dr. Fernanda Pérez (Departamento de Ecología, Facultad de Ciencias Biológicas, Pontificia Universidad Católica de Chile, Santiago de Chile). Specimens have been deposited in the herbarium of Departamento de Ecología y Botánica, Pontificia Universidad Católica de Chile, Santiago de Chile (reference number 2006-1/2-S).

Flowers were freeze-dried and sent to Spain for the analyses. The samples were kept in desiccators until analysed.

\subsection{Standards}

Delphinidin and petunidin 3-O-glucosides were purchased from Polyphenols Labs (Sandnes, Norway). Caffeic, $p$-coumaric and ferulic acids ( $E$-isomers) were obtained from Sigma-Aldrich Chemie (Steinheim, Germany) as well as $\mathrm{D}(+)$-glucose, $\mathrm{D}(+)$-galactose and $\alpha$-L-rhamnose.

\subsection{Sample preparation}

Taking into account that the pigment distribution along the petals of the flowers of both species is not homogeneous and that the upper petal also possess yellow pigmentation complete corollas were selected for the analyses. Furthermore, three different extractions were carried out for each species in order to obtain representative quantitative results. For each extraction, ca. $100 \mathrm{mg}$ was weighed and macerated with $100 \mathrm{~mL}$ of $\mathrm{MeOH}: \mathrm{HCl} 0.5 \mathrm{~N}$ (95:5) at $-20^{\circ} \mathrm{C}$ overnight. The extracts were filtered on a Büchnel funnel and the petals were re-extracted twice with the same solvent and sonicated during $30 \mathrm{~min}$. The methanolic extracts were gathered and concentrated under vacuum in a rotary evaporator at $35^{\circ} \mathrm{C}$ and once the methanol was eliminated the pigments were taken to a final volume of $20 \mathrm{~mL}$ with acidified water ( $\mathrm{pH} 1.4, \mathrm{HCl}$ ). For the quantitative purpose, each extract of each species was analysed by HPLC-DAD in triplicate ( 9 samples per species). The samples were filtered through a $0.50 \mu \mathrm{m}$ Dismic $^{\circledR}-13$ JP syringe filter unit (Toyo Roshi Kaisha, Ltd., Japan) prior to the analyses. Concentrations $(\mathrm{mg} / \mathrm{mL})$ of individual compounds were calculated from the area of their corresponding peaks in the chromatograms recorded at $520 \mathrm{~nm}$ using two different calibration curves, one built with delphinidin 3-O-glucoside (for delphinidin-derived pigments, compounds 1, 2, 3, 4, 7 and 11) and the other built with petunidin 3-O-glucoside (for petunidin-derived pigments and other methoxylated anthocyanins, compounds $5,8,9,10,12,13 / 14,15$. Compound $\mathbf{6}$ co-eluted with compound $\mathbf{7}$ and both were quantified jointly). Concentrations of individual compounds in dried petals were then calculated $(\mathrm{mg} / \mathrm{g})$ taking into account the initial amount of petals weighed. For the qualitative analyses, only one of the 9 available samples for each species was analysed by HPLC-DADMS. In order to minimize the interferences of other phenolic compounds in the identification of the pigments, these two selected samples were purified by solid phase extraction as follows: the samples were applied onto a Waters C-18 Sep-Pak ${ }^{\circledR}$ (500 mg) cartridge (Millipore Corp., Milford, MA, USA), previously activated with methanol: $\mathrm{HCl} 0.1 \mathrm{~N}$ (95:5) and equilibrated with ultrapure water. Anthocyanins were retained. Sugars and acids were eluted with water, some flavanols and flavonols were eluted with ethyl acetate and the anthocyanins were finally recovered with methanol: $\mathrm{HCl} 0.1 \mathrm{~N}(95: 5)$. The methanol was evaporated in a rotary evaporator and the pigments taken to a known volume with acidified water $(\mathrm{pH} \mathrm{1.4,} \mathrm{HCl})$. These samples were also filtered before the injection.

\subsection{Alkaline hydrolysis of anthocyanins}

Alkaline hydrolysis was carried out in the S. grahamii anthocyanin fraction obtained after purification of the initial extract with 
solid phase extraction and also in the acylated anthocyanins isolated from this extract in order to confirm the identities of the acids involved in the acylation and to prepare the sample for subsequent acid hydrolysis. Four millilitres of the sample was introduced in a screw-cap test tube. $10 \%$ aqueous $\mathrm{KOH}$ were then added until the colour became blue, nitrogen was flushed and the tube was capped and maintained in the dark at room temperature for $10 \mathrm{~min}$. The soln. was neutralised with $3 \mathrm{~N} \mathrm{HCl}$ until the recovery of red colouration. The separation between phenolic acids and anthocyanins was performed with ethyl ether. The organic phase, containing the phenolic acids, was concentrated under vacuum and re-dissolved in $\mathrm{MeOH}: \mathrm{H}_{2} \mathrm{O}$ (10:90). The aqueous phase was also concentrated under-vacuum and re-dissolved in acidic water $(\mathrm{pH} \mathrm{1,} \mathrm{HCl})$. Both phases were analysed by HPLC-DAD.

\subsection{Acid hydrolysis of anthocyanins}

Ten millilitres of $3 \mathrm{~N} \mathrm{HCl}$ was added to the aqueous phase resulting from the alkaline hydrolysis in a screw-cap test tube, flushed with nitrogen and capped. The tube was placed at $100{ }^{\circ} \mathrm{C}$ for $60 \mathrm{~min}$ and then cooled in an ice bath. The hydrolysate was purified using a Waters C-18 Sep-Pak ${ }^{\circledR}$ cartridge previously activated with methanol: $\mathrm{HCl} 0.1 \mathrm{~N}(95: 5)$ and equilibrated with ultrapure water. The sample was loaded onto the cartridge and ultrapure water was first added in order to elute the sugars released from the pigments. This fraction was concentrated under vacuum and then analysed by HPLTC.

The same protocol was employed in the acid hydrolysis of the isolated pigments.

\subsection{HPTLC analyses}

The sugar-rich fraction of the extract and those of the isolated pigments were analysed by HPLTC following the procedure described by di Paola-Naranjo et al. (2004). Silica Gel 60 HPTLC plates $(5 \times 5 \mathrm{~cm}$, Merck, Germany) were used for the analysis. Plates were impregnated with $0.02 \mathrm{M}$ boric acid and activated at $100{ }^{\circ} \mathrm{C}$ for $30 \mathrm{~min}$ before use. Then, the sample was spotted in the plate $(10 \mu \mathrm{L})$ as well as the standards $(5 \mu \mathrm{L}, 1 \mathrm{mg} / \mathrm{mL}$ aq. soln. of $\mathrm{D}(+)$-glucose, $\mathrm{D}(+)$-galactose and $\alpha$-L-rhamnose) and the chromatography was developed horizontally with $n$-propanol:water (7:1, $\mathrm{v} / \mathrm{v}$ ). After air-drying the plates, the sugar spots were revealed as described by Toba and Adachi (1978) by spraying a diphenylamine-aniline reagent (4 g diphenylamine, $4 \mathrm{~mL}$ aniline, $20 \mathrm{~mL}$ phosphoric acid $85 \%$ and $200 \mathrm{~mL}$ acetone), allowing to stand until they were nearly dry and heating at $80^{\circ} \mathrm{C}$ for $20 \mathrm{~min}$.

\subsection{Isolation of pigments}

Some of the major pigments were isolated from S. grahamii in order to carry out further alkaline and acid hydrolyses and HPTLC to confirm the identities proposed from the results of the HPLCDAD-MS analyses. The same equipment (chromatograph, column) as that employed in the HPLC-DAD analyses was used for the isolation of the compounds. The sample was concentrated at the head of the column, by injecting $100 \mu \mathrm{L}$ of the sample 20 times consecutively ( 2 min between injections) and using isocratic 100\% ultrapure water (solvent $\mathrm{C}$ ) at a flow rate of $0.5 \mathrm{~mL} / \mathrm{min}$ during the whole injection period. After the loading step, the elution and separation of the compounds were achieved by using solvents $\mathrm{A}, \mathrm{B}$ and $C$ with the following gradient: isocratic $100 \%$ C for $5 \mathrm{~min}$, from $100 \%$ C to $85 \%$ A and 15\% B for 25 min, isocratic $85 \%$ A and $15 \%$ B for $20 \mathrm{~min}$, from $85 \% \mathrm{~A}$ and $15 \% \mathrm{~B}$ to $80 \% \mathrm{~A}$ and $20 \% \mathrm{~B}$ for $5 \mathrm{~min}$, isocratic $80 \% \mathrm{~A}$ and $20 \%$ B for $15 \mathrm{~min}$, from $80 \% \mathrm{~A}$ and $20 \%$ B to $50 \% \mathrm{~A}$ and $50 \%$ B for 5 min and from $50 \% \mathrm{~A}$ and $50 \%$ B to $50 \%$ B and $50 \%$ C for $5 \mathrm{~min}$. The flow rate was $0.5 \mathrm{~mL} / \mathrm{min}$. Spectra were recorded from 220 to $600 \mathrm{~nm}$. Isolated compounds were collected through the UV-cell outlet.

\section{Acknowledgements}

Thanks are due to Junta de Castilla y León (group GR133) for financial support. The authors also want to thank Dr. Fernanda Pérez (Departamento de Ecología, Facultad de Ciencias Biológicas, Pontificia Universidad Católica de Chile, Santiago de Chile) for the collection and the identification of the plant material.

\section{References}

Alcalde-Eon, C., Escribano-Bailón, M.T., Santos-Buelga, C., Rivas-Gonzalo, J.C., 2006 Changes in the detailed pigment composition of red wine during maturity and ageing. A comprehensive study. Anal. Chim. Acta 563, 238-254.

Alcalde-Eon, C., Saavedra, G., de Pascual-Teresa, S., Rivas-Gonzalo, J.C., 2004a. Liquid chromatography-mass spectrometry identification of anthocyanins of isla oca (Oxalis tuberosa, Mol.) tubers. J. Chromatogr. A 1054, 211-215.

Alcalde-Eon, C., Saavedra, G., de Pascual-Teresa, S., Rivas-Gonzalo, J.C., 2004b. Identification of anthocyanins of pinta boca (Solanum stenotomum) tubers. Food Chem. 86, 441-448.

Andersen, Ø.M., Jordheim, M., 2006. The anthocyanins. In: Andersen, Ø.M. Markham, K.R. (Eds.), Flavonoids: Chemistry, biochemistry, and Applications. CRC Press, Taylor \& Francis Group, Boca Ratón (USA), pp. 471-551.

Ando, T., Saito, N., Tatsuzawa, F., Kakefuda, T., Yamakage, K., Ohtani, E., Koshi-ishi, M., Matsusake, Y., Kokubun, H., Watanbe, H., Tsukamoto, T., Ueda, Y. Hashimoto, G., Marchesi, E., Asakura, K., Hara, R., Seki, H., 1999. Floral anthocyanins in wild taxa of Petunia (Solanaceae). Biochem. Syst. Ecol. 27 623-650.

Ando, T., Takahashi, M., Nakajima, T., Toya, Y., Watanabe, H., Kokubun, H. Tatsuzawa, F., 2004. Delphinidin accumulation is associated with abnormal flower development in petunias. Phytochemistry 65, 2219-2227.

André, C.M., Oufir, M., Guignard, C. Hoffmann, L., Hausman, J.F., Evers, D. Larondelle, Y., 2007. Antioxidant profiling of native Andean potato tubers (Solanum tuberosum L.) reveals cultivars with high levels of $\beta$-carotene, $\alpha$ tocopherol, chlorogenic acid, and petanin. J. Agric. Food Chem. 55, 1083910849.

Cretton, S., Bartholomeusz, T.A., Humam, M., Marcourt, L., Allenbach, Y., Jeannerat, D., Muñoz, O., Christen, P., 2011. Grahamines A-E, cyclobutane-centered tropane alkaloids from the aerial parts of Schizanthus grahamii. J. Nat. Prod. 74, 2388-2394

Dangles, O., Saito, N., Brouillard, R., 1993. Anthocyanin intramolecular copigment effect. Phytochemistry 34, 119-124.

di Paola-Naranjo, R.D., Sánchez Sánchez, J., González-Paramás, A.M., Rivas-Gonzalo, J.C., 2004. Liquid chromatographic-mass spectrometric analysis of anthocyanin composition of dark blue bee pollen from Echium plantagineum. J. Chromatogr. A 1054, 205-210.

Fossen, T., Cabrita, L., Andersen, Ø.M., 1998. Colour and stability of pure anthocyanins influenced by $\mathrm{pH}$ including the alkaline region. Food Chem. 63 , 435-440.

Fossen, T., Øvstedal, D.O., Slimestad, R., Andersen, Ø.M., 2003. Anthocyanins from a Norwegian potato cultivar. Food Chem. 81, 433-437.

George, F., Figueiredo, P., Toki, K., Tatsuzawa, F., Saito, N., Brouillard, R., 2001 Influence of trans-cis isomerisation of coumaric acid substituents on colour variance and stabilisation in anthocyanins. Phytochemistry 57, 791-795.

Giusti, M.M., Rodríguez-Saona, L.E., Griffin, D., Wrolstad, R.E., 1999. Electrospray and Tandem mass spectroscopy as tools for anthocyanin characterization. J Agric. Food Chem. 47, 4657-4664.

Harborne, J.B., 1958. Spectral methods of characterizing anthocyanins. Biochem. J. $70,22-28$.

Harborne, J.B., Grayer, R.J., 1994. Flavonoids and insects. In: Harborne, J.B. (Ed.), The Flavonoids: Advances in Research Since 1986. Chapman \& Hall, London, pp. 589-618

Harborne, J.B., Smith, D.M., 1978. Correlations between anthocyanin chemistry and pollination ecology in the Polemoniaceae. Biochem. Syst. Ecol. 6, 127-130.

Ichiyanagi, T., Kashiwada, Y., Shida, Y., Ikeshiro, Y., Kaneyuki, T., Konishi, T., 2005. Nanusin from eggplant consist of cis-trans isomers of Delphinidin 3-[4- $(p-$ coumaroyl)-L-rhamnosyl (16)glucopyranoside]-5-glucopyranoside. J. Agric. Food Chem. 53, 9472-9477.

Miller, R., Owens, S.J., Rørslett, B., 2011. Plants and colour: Flowers and pollination. Opt. Laser Technol. 43, 282-294.

Mol, J., Grotewold, E., Koes, R., 1998. How genes paint flowers and seeds. Trends Plant Sci. 3, 212-217.

Peña, R.C., Muñoz, O., 2002. Phylogenetic relationships in the genus Schizanthus (Solanaceae). Biochem. Syst. Ecol. 30, 45-53.

Pérez, F., Arroyo, M.T.K., Medel, R., Hershkovitz, M.A., 2006. Ancestral reconstruction of flower morphology and pollination systems in Schizanthus (Solanaceae). Am. J. Bot. 93, 1029-1038

Pérez, F., Arroyo, M.T.K., Medel, R., 2007. Phylogenetic analysis of floral integration in Schizanthus (Solanaceae): Does pollination truly integrate corolla traits? J. Evol. Biol. 20, 1730-1738. 
Pérez, F., Arroyo, M.T.K., Armesto, J.J., 2009. Evolution of autonomous selfing accompanies increased specialization in the pollination system of Schizanthus (Solanaceae). Am. J. Bot. 96, 1168-1176.

Pérez, F., Spencer, P., Cienfuegos, A., Suárez, L., 2011. Microsatellite markers for the high Andean species Schizanthus hookeri and S. grahamii (Solanaceae). Am. J. Bot. 98, e114-e116.

Rodríguez-Gironés, M.A., Santamaría, L., 2004. Why are so many bird flowers red? PLoS Biol. 2, e350.

Rodríguez-Saona, L.E., Giusti, M.M., Wrolstad, R.E., 1998. Anthocyanin pigment composition of red-fleshed potatoes. J. Food Sci. 63, 458-465.

Saito, N., Harborne, J.B., 1992. Correlations between anthocyanin type, pollinator and flower colour in the labiatae. Phytochemistry 31, 3009-3015.
Strack, D., Wray, V., 1994. The anthocyanins. In: Harborne, J.B. (Ed.), The Flavonoids: Advances in Research Since 1986. Chapman \& Hall, London, pp. 1-22.

Tatsuzawa, F., Ando, T., Saito, N., Kanaya, T., Kokubun, H., Tsunashima, Y., Watanabe, H., Hashimoto, G., Hara, R., Seki, H., 2000. Acylated delphinidin 3-rutinoside-5glucosides in the flowers of Petunia reitzii. Phytochemistry 54, 913-917.

Toba, T., Adachi, S., 1978. Specific colour reaction for the detection of 1,2-linked reducing disaccharides on paper and thin-layer chromatograms. J. Chromatogr. $154,110-112$

Wilbert, S.M., Schemske, D.W., Bradshaw Jr., H.D., 1997. Floral anthocyanins from two monkeyflower species with different pollinators. Biochem. Syst. Ecol. 25, 437-443. 